\title{
RAPID DATA COLLECTION FOR THE AUDIT OF MONUMENTS \& MEMORIALS IN SOUTH AFRICA
}

\author{
C. Jackson ${ }^{\mathrm{a}}$, M. Nkhasi-Lesaoana ${ }^{\mathrm{a}}$, L. Mofutsanyana ${ }^{\mathrm{a}}$
}

\begin{abstract}
a South African Heritage Resources Agency (SAHRA), 111 Harrington Street Cape Town 8000, South Africa -
\end{abstract} cjackson@sahra.org.za, lmofutsanyana@sahra.org.za,mnkhasi@sahra.org.za

KEY WORDS: Heritage Inventories, South Africa, SAHRIS, KOBOtoolbox, Data Collection

\begin{abstract}
:
The tradition of memorialising people and events through physical constructions such as statues and monuments like in many countries, has shaped the public space of a modern South Africa. Considering the colonial and apartheid history of South Africa, these physical markers, often uncontextualized, continue to maintain positions of prominence within the modern streetscape.
\end{abstract}

Since the turn of the democratic era in South Africa, a pressing need has existed to assess the impact of the markers on the heritage landscape of the country. An endeavour made more difficult by a lack of a comprehensive inventory of these resources across the country.

The National Audit of Monuments and Memorials (NAMM) was designed to address this gap through a full national survey of monuments and memorials, conducted under the auspices of a job creation stimulus package designed to create short term employment in the wake of the economic fallout from the Covid-19 pandemic. Undertaking this project under this funding mechanism required that all phases of the project be undertaken within a six-month period.

The compressed timeframes associated with this project required an approach that could support a level of fluidity to address the challenges of undertaking a project of this nature, whilst ensuring that the data collected by field surveyors can be monitored and included in the inventory of the national estate in an effective manner.

The aim of this paper is to discuss and showcase the tools and workflows used to roll out and manage the large-scale national audit of monuments and memorials across South Africa.

\section{INTRODUCTION}

The issue of Monuments and Memorials in South Africa, especially statues, and the need to reassess how South African heritage is presented has been a pressing concern from the turn of the democratic dispensation in 1994. Noting this, a principal concern of the South African Heritage Resources Agency (SAHRA) and the Department of Sport, Arts and Culture (DSAC) is the identification of these monuments and memorials throughout the country.

With renewed public pressure brought forth by the Rhodes Must Fall movements, widespread discussions on decolonisation of colonial structures in modern day South Africa, and recently in the wake of global Black Lives Matter protest action, the need to identify and readdress the impact these markers have on the fabric of a post-apartheid South Africa became more imperative (Kiewit, 2020).

This National Audit of Monuments and Memorials (NAMM) was designed to provide the first intervention, through a national survey aimed at identifying these markers. This project was made possible through funding established by the Presidency of the Republic of South Africa under the auspices of a job creation stimulus package designed to create short term employment in the wake of the economic fallout from the Covid-19 pandemic (Republic of South Africa, 2020). Whilst funding was provided for the employment of 260 participants, this source of funding presented its own challenges as it required that all planning and implementation be completed within a six-month period.

The compressed timeframes associated with this project required an approach that could support a level of fluidity to address the challenges of undertaking a project of this nature, whilst ensuring that the data collected by field surveyors can be monitored and included in the inventory of the national estate in an effective manner.

To facilitate this, it was decided that a technological approach should be taken to minimise post field work data capture, alert the project management team to data quality issues, and provide a more rapid solution for reporting.

\section{MONUMENTS \& MEMORIALS}

\subsection{Legal standing of Monuments \& Memorials in South Africa}

The National Heritage Resources Act, act 25 of 1999 (NHRA), South Africa's legislation for the protection of the country's heritage, specifically includes monuments \& memorials within the national estate and thus affords them protection through the mechanisms employed within the legislation. Specifically 
public monuments and memorials are afforded automatic protection under a mechanism known as the "Heritage Register" which is carried out generally by the Local Authority (Municipality), either by means of a Planning Scheme or Bylaws, unless the significance of the resource is considered to warrant its management at a higher tier of government level and declared to be either a Provincial or National Heritage Site.

The NHRA, through its definitions, generally provides some clarity on how certain types of heritage resources are classified (eg: archaeological, palaeontological, graves, etc...). In the case of public monuments and memorials, the NHRA provides the following;

"public monuments and memorials" means all monuments and memorials -

(a) erected on land belonging to any branch of central, provincial or local government, or on land belonging to any organisation funded by or established in terms of the legislation of such a branch of government; or

(b) which were paid for by public subscription, government funds, or a public-spirited or military organisation, and are on land belonging to any private individual;" (South Africa, Dept. of Arts and Culture, 1999)

Whilst addressing identification in terms of locality and public intent, it remains silent on their nature. An issue compounded by previous heritage legislation applying the term "National Monuments" to all resources formally declared under the National Monuments Council Act, act 28 of 1969. This has resulted in the necessity of turning to other sources for appropriate definition. In 2003 SAHRA undertook a survey of public monuments and memorials within the three capitals, where the project leader turned to the New Oxford Dictionary of English and the Collins English Dictionary to establish a common understanding under which to identify these resources, namely as "structures and immovable objects which commemorate a person, group, organization or event and are situated on public open spaces" (Marx, 2003).

This definition was further expanded upon in the Guidelines for Public Monuments and Memorials produced by Heritage Western Cape, as follows;

"structures, memorials, statues erected on public or privately owned space (including their plinths) and other immovable objects which commemorate a person, group, organization or event and are situated on public or private open spaces." (HWC, 2015)

Whist this amendment addresses the acknowledgement of public monuments and memorials existing on privately owned land, a need still exists for a more prescriptive identification mechanism that assists various parties in identifying them, as the experience with the NAMM has shown that a common understanding regarding what is a monument, or a memorial differs from person to person.

The debate around the presence of monuments and memorials in public spaces has largely centred around statues as a specific form of memorialisation, however the act of constructing physical monuments and memorials takes many different constructed forms such as obelisks, plaques, and open spaces. As a result, a myopic focus on statues and a lack of understanding the various forms these constructs may take serves only to undermine their identification and protection, thus with the context of the NAMM focus was not solely placed on statues but was inclusive of the various constructed forms of memorialisation.

\subsection{Transformation of the Heritage Landscape and Memorialisation in the South African context}

The act of memorialising people and events through the construction of physical markers such as statues and monuments is a long tradition, one which leaves a lasting impact on the streetscape of a country. In South Africa considering the colonial and apartheid history, these physical markers continue to serve as reminders of a painful past imposed by some of the leaders of the era that shaped the Country's oppressive and exclusive history.

The transformation of the heritage landscape is a call for public symbols; representation of the heritage national estate that is in line with the Constitution of South Africa. That is the view that the statues, place names and other form of symbolism in public spaces needs to be in line with the values and spirit of a nonracial, democratic and inclusive South Africa. Since the dawn of the democratic dispensation in 1994, this implies the need to reassess how the collective South African heritage is representative of the country's diverse cultures (Rankin, 2013). This view is in line with the policies of the government of the day which called for integration of apartheid and colonial history markers in the new South Africa. As a result, efforts, where possible, went into the reinterpretation and reforming of these makers (Marschall, 2006; Rankin, 2013). In 2015 there was a move towards the repositioning and relocation of the statues associated with the country's oppressive, apartheid history which escalated to the removal of the Rhodes Statue at the University of Cape Town resulting from the student's "Rhodes must fall" movement (Marschall, 2017). To address the concerns regarding offensive public symbols and markers in a way that promotes social cohesion and nation building it was important that an audit of all statues; memorials and monuments is undertaken at a national scope.

As the national body responsible for heritage management, the South African Heritage Resources Agency (SAHRA) was tasked with a country-wide survey, aimed specifically at recording, and documenting the locations of monuments \& memorials, with the view to further assess their significance.

\subsection{Inventories of Monuments and Memorials}

SAHRA is required to compile and maintain an inventory of the national estate, meaning all heritage resources across the country of cultural significance.

This NHRA institutes a mechanism for the formulation of heritage inventories which follows a three-tier governance structure. This requires that all local authorities (municipalities) compile an inventory of conservation worthy properties within their jurisdiction and submit this to the provincial authority for inclusion in the provincial heritage register, who in turn must submit this to SAHRA for inclusion in the inventory of the national estate (for a further discussion on the three-tier system of heritage management see: Jackson et al, 2019).

The success of this is predicated on the functioning of the threetier system. At present, this model is not functional, with institutional problems plaguing many of the provincial authorities (Jackson et al, 2019). As a result, SAHRA lacked a 
comprehensive inventory of monuments and memorials across the country.

In previous years there were attempts to address this with a SAHRA initiated survey of the three capital cities in 2003 (Marx, 2003), a localised survey by the City of Cape Town in 2009 (O’Donoghue, 2009), and a survey of the Free State Province conducted but the Free State Provincial Heritage Resources Authority (FSPHRA, 2016).

Whilst these surveys were vital early steps, considering the localised nature of these surveys, and a lack of location data in the case of the Free State survey, the need to undertake a largescale audit and inventorisation exercise was required to expand the limited inventory of 329 monuments and memorials which SAHRA had existing records of.

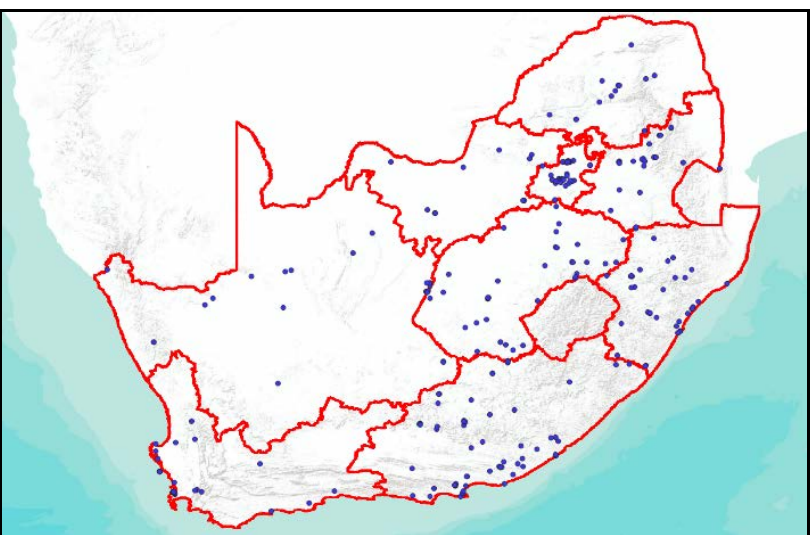

Figure 1: Map of known monuments and memorials in South Africa prior to the NAMM.

\section{NATIONAL AUDIT OF MONUMENTS \& MEMORIALS}

\subsection{The Presidential Employment Stimulus Programme (PESP)}

The Presidential Public Employment Stimulus Programme (PESP) is a programme instituted by the South African government in response to the prevailing impact of the COVID19 pandemic on jobs and livelihoods of South Africans. The stimulus package is aimed at the creation of short-term job opportunities, as a form of alleviating the prolonged impact of the pandemic (Republic of South Africa, 2020). Undertaking this largescale audit of South Africa's national estate as a project within the PESP created employment opportunities for unemployed people.

The national directives on the PESP required that approved projects would need to be undertaken within a set period of six months, despite the challenge of rolling out a national project within these timeframes, amidst the Covid-19, rapid project implementation planning commenced. These timeframes were compressed further as delays in final project approval and budgetary allocation resulted in the need readjust timelines and strategies, with a final effective period of four months to physically survey the country. Due to highly limited timeframes that allowed very little time for project scoping and planning, it was necessary for a specific approach that would allow rapid data collection in the field to be developed.

\subsection{Aims of the National Audit}

The National Audit of Monuments \& Memorials (NAMM) - A Prerequisite to Heritage Landscape Transformation Programme serves as the intervention to provide an inventory of Monuments \& Memorials across the country needed for the Heritage Landscape Transformation Programme.

Broadly, the NAMM aimed to

1. Identify monuments and memorials across the country.

2. Photograph and record locations of identified resources.

3. Capture basic information that can be used as a basis for assessment of significance.

4. provide employment opportunities to unemployed youth as part of the Covid-19 economic stimulus.

5. Provide the baseline for decision making within the broader Heritage Landscape Transformation Programme

\section{DIGITAL APPROACH FOR RAPID DATA COLLECTION}

The scope and timelines associated with the NAMM presented a new challenge for SAHRA in terms of how the data would be collected, monitored, and processed. Whilst previous work had been conducted on the construction of surveying toolkits and recording forms, these were based on a traditional pen-andpaper based approach. The usage of a pen-and-paper system would require the establishment of an effective network for the printing, distribution and digitisation of completed survey forms. The formulation of this network was not a feasible objective within this project due to time constraints and the lack of accessibility to these resources, especially considering the distance and travel time between major centres population and more rural towns. Additionally, this method would necessitate an extensive period of data capture towards the end of the project, thereby limiting functional fieldwork time. Dauenhauer et al. (2019) further notes the difficulties encountered during the processing of physical paper-based surveys, which include; the legibility of written responses, and standardisation of data entry. Furthermore, as a survey of this nature is inherently spatial, the ability to collect good quality location data is a critical success factor.

The ongoing COVID-19 pandemic presented its own challenges in terms of the ability to provide comprehensive training opportunities. Whilst some of the challenges noted by Dauenhauer et al. (2019) are possible to be mitigated through training, the ability to provide said training was a limiting factor due to restrictions imposed on gatherings and a lack of access to internet in some areas. As full fieldwork time needed to take priority, and training opportunities were limited, a digital, and rapidly deployable ecosystem was needed that could serve to mitigate the noted limitations and provide appropriate remote, and real time, oversight.

\subsection{Digital Surveys}

Based on the limitations noted above, a solution was required that addressed the following areas;

1. Rapidly deployable

2. Standardisation and validation of data collection

3. Remote oversight and quality review 
4. Ease of reporting

5. Offline capture of data

6. Photography of finds

7. Geolocation capture

8. Ease of integration with the inventory of the national estate

As noted by Dauenhauer et al. (2019), a digital approach provides higher quality data to be collected at a greater pace, as well as providing a mechanism for mitigating the limitations noted in the preceding discussions.

Whilst SAHRA has been managing heritage in the digital space for a number of years through the South African Heritage Resources Information System (SAHRIS), it was not designed to facilitate field data collection in the manner required by this project. As a purely online web-based platform this further precludes its direct utility in areas where internet access is limited.

Whilst there are numerous options available for data collection (see Bokonda et al, 2020), the KoBoToolbox was selected for this project due to ease of configuration, intuitive interface, ability to analyse and moderate collected data within the interface, and a mobile application that allows for offline data capture.

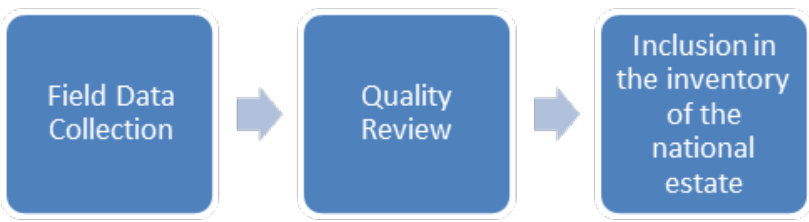

Figure 2: Basic data flow

Though this approach does address the requirements noted above, it also introduces a new set of challenges relating to the acquisition, distribution, and configuration of equipment. These will be discussed further in the discussions around design and implementation.

4.1.1 KoBoToolbox: The KoBoToolbox was used as the primary data collection server for the project. The system is purpose built as a specialist package for field data collection. It allows for the rapid development and deployment of data collection forms and provides a ready to use mobile app that can be used both on and offline, and then synced back to the data collection server (KoBoToolbox, n.d.-a.). This tool further provides an API which allows the collected data to be synced to the South African Heritage Resources Information System (SAHRIS) for incorporation in the inventory of the national estate (KoBoToolbox, n.d.-a.).

KoBoToolbox is free, open source, and able to be deployed within SAHRA's own server environment to ensure security.

The management interface allows for real time tracking of returned data, and the ability to moderate data submitted prior to the automated transfer to the SAHRIS database. This allowed the integrity of the data to be monitored throughout the course of the project and alert the management team to any potential need for intervention on data quality in real time.

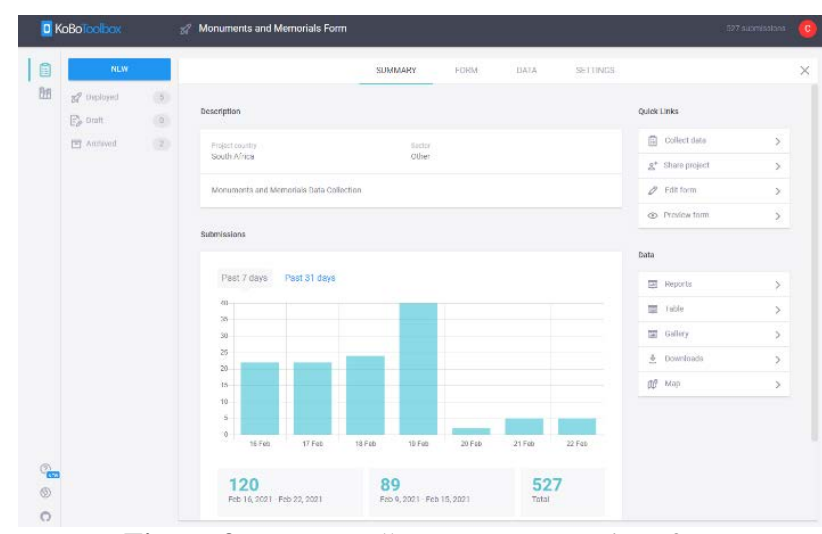

Figure 3: KoBoToolbox management interface

4.1.2 KoBoCollect: KoBoCollect is an OpenDataKit based mobile application, that allows data capture forms created in KoBoToolbox to be completed on any android based mobile device (KoBoToolbox, n.d.). It is able leverage the capabilities of any modern smartphone to take photographs and GPS coordinates without the need for additional equipment such as cameras or handheld GPS. The ability of the application to work offline and later sync to the primary server was a further consideration, as much of rural South Africa lacks adequate mobile network coverage for an always connected service.
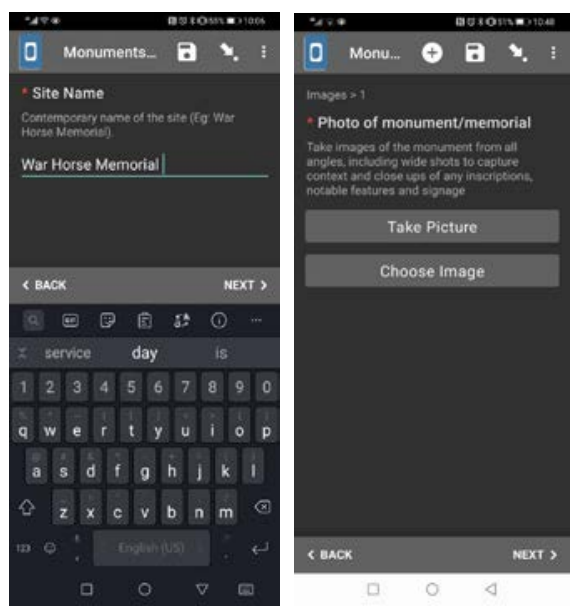

Figure 4: Example of KoBoCollect interface

4.1.3 SAHRIS: The South African Heritage Resources Information System (SAHRIS) is a free, open-source and webbased content system designed to fulfil stipulations of section 39 of the National Heritage Resources Act, No. Act 25, of 1999. Section 39 mandates SAHRA, for purposes of consolidation and coordination of heritage information, to compile and maintain a database of conservation worthy heritage resources into an inventory of the National Estate (South Africa, Department of Arts and Culture, 1999).

Publicly launched in 2013, SAHRIS functions as a national repository of heritage sites, a collection management system for objects and an integrated heritage management system which allows for complete heritage management across all spheres of governance (Wilshire, 2013). Through these functions SAHRIS facilitate the standardization for recording heritage information; general principles for governing heritage resources; promoting good management across the three governance levels; encourages civil society to nurture and conserve their heritage resources through active identification and recording. 


\subsection{Design and Implementation}

The data intended for capture through this survey is broken into 5 key areas, these being; surveyor details, locality, imagery, physical descriptors, and commemorative intent.

Acknowledging the inexperience and limited training of the field surveyors, the survey was specifically designed to enforce collection of critical information such as locality, imagery, and physical descriptions, whilst allowing the flexibility to capture the more qualitative information on commemorative intent should that information be available through inscriptions of interpretive signage on or near the site.

This flexibility was specifically built into the survey due to ongoing and widespread issues of vandalism and theft of material from monuments and memorials. In many cases the traditionally bronze plaques that contained interpretive information have been either removed for protection of stolen, thus resulting in a lack of onsite information available to field surveyors.

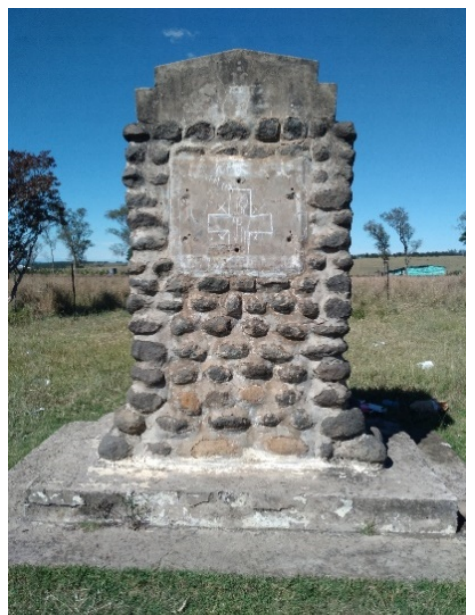

Figure 5: Battle of Draaibosch Memorial with interpretative plaque removed.

4.2.1 Tailoring the solution: The KoBoToolbox allows for recording forms to be developed in two ways. Firstly, directly through the interface using a simple system of predetermined question types (eg: text entry, multiple select, etc...), which can then be customised with validation criteria and skip logic (KoBoToolbox. n.d.-c). Alternatively, the form can be developed within a spreadsheet application such as MS Excel or Google Sheets, and then uploaded to the KoBoToolbox server in XLSform format (KoBoToolbox. n.d.-b). The mandatory response criteria allowed us to ensure that locality, imagery, and physical descriptions were captured in all recording instances and allowing submission of the collected data even where additional information was not available to the field recorder.

The configuration of the android based mobile phones supplied to the enumerators initially presented a challenge due to the number of devices (105), and the need to provide appropriate authentication for the teams in each of the nine provinces. This was overcome using a Quick Response (QR) Code configured with the authentication information for each of the provinces (KoBoToolbox. n.d.-e). After installation of the KoBoCollect app on each device, the QR code was able to be scanned and would automatically configure the app to sync with the primary KoBoToolbox server, apply default metadata per province and institute an admin lockout so that the settings could not be changed accidentally during use.

To begin operating, the field surveyor only required access to the internet for a brief period to download the collection form from the server. The form could then be completed offline and stored until the surveyor was able to connect to a mobile network, at which point all collected data, inclusive of images, would be synced to the server for further processing.

Within the primary KoBoToolbox interface, coordinators within each of the provinces were given access rights to review, edit and validate submissions made by the field surveyors as they are synced (KoBoToolbox. n.d.-g).

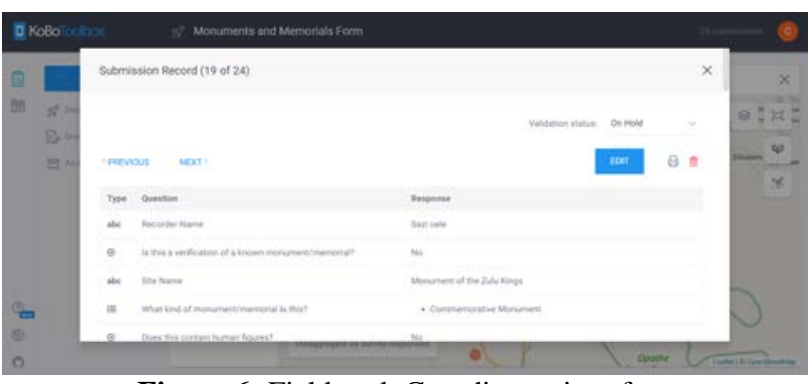

Figure 6: Fieldwork Coordinator interface

Due to the spatial nature of this project, the KoBoToolbox allows for the visualisation of received data through a simple map interface (KoBoToolbox. n.d.-f). This allowed SAHRA to track the progress of the field teams in near real time through their submissions and thereby identify regions of slower deployment and the extent of geographic coverage.

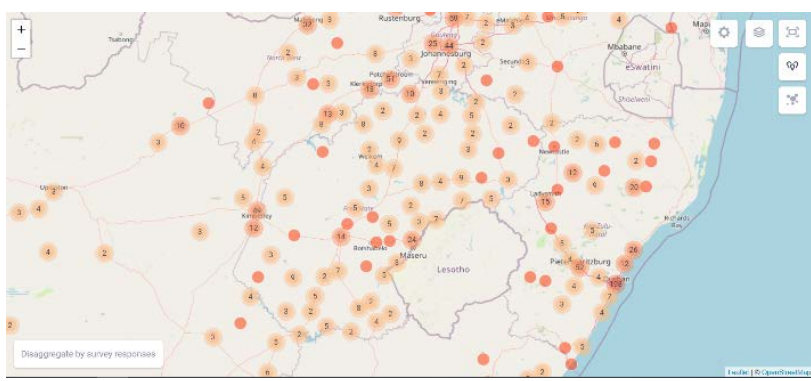

Figure 7: Online map interface showing survey submissions.

4.2.2 Integration between systems: A key component of the overall flow of data was the ability to easily integrate data verified by the Fieldwork Coordinators into SAHRIS as the system that hosts the inventory of the national estate. Whilst KoBoToolbox provides an easy method of exporting data from the collection server to excel, this would have required manual intervention to upload the exported file to SAHRIS, additionally the media attachments associated with the records would require manual upload to each of the newly created inventory records. While four employment opportunities within the project was allocated to data moderation within SAHRIS, undertaking the manual import would have resulted in a large time allocation be dedicated to this process as opposed to focussing on performing a second level review of imported records.

KoBoToolbox provides an Application Programming Interface (API) that allows data stored in the KoBoToolbox database to be retrieved and written to the SAHRIS database (KoBoToolbox. n.d.-h). 
This allowed for the setup of an automated transfer of data based on the validation status set by the Fieldwork Coordinator. At regular intervals, SAHRIS would scan through the data assets on KoBoToolbox and write newly approved records to the SAHRIS database together with associated media files.

Overall, this approach proved to be effective in ensure that there was a rapid flow of data from the field through to the inventory.

There were however concessions that needed to be accepted within this process due to the structured data stored on SAHRIS versus the easiest methods of recording in the field. Minor thesauri employed on SAHRIS were easily incorporated into the recording form, however major authority files were not. An example of this are the authority files for People and Organisations. Within the SAHRIS ecosystem, Persons and Organisations are stored as unique entities with unique keys for identification and reference. Considering the size of the authority files, and various possible permutations of already captured names that may be encountered in the field, it was decided that attempting to fully integrate would present a risk of creating duplicate entries, or worse, creating inaccurate references between the authority file and the site being recorded. To address this, the data was imported in an unstructured text format, and reliance was placed on the moderation staff to either link existing records or create new entries based on the survey data, before escalating the records for further desktop research.

Ultimately the workflow between the various systems took the following form.

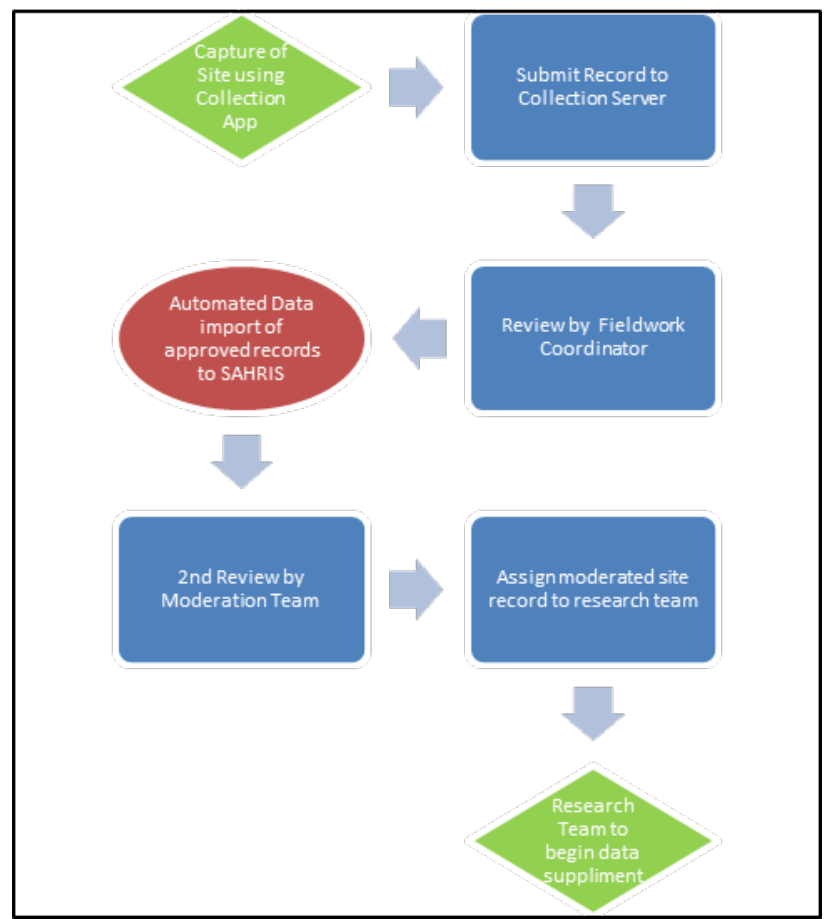

Figure 8: Simplified data workflow

\section{OUTCOMES}

\subsection{Achievements and Results}

The implementation of the discussed systems and workflows ultimately achieved the aims of the project and was able to be rolled out rapidly. The total time taken between approval of the data capture ecosystem to roll out of testing was eight days. This is inclusive of server resource allocation, installation of the KoBoToolbox ecosystem and the creation of the recording form. A benefit of having undertaken this process is now SAHRA will be able to utilise the same ecosystem for future projects, either initiated by SAHRA or by other heritage authorities, with a fully customised data collection form with a lead time of as little as a day (depending on the complexity of the data to be captured). Active testing has already been conducted with the use of the platform to facilitate the site monitoring activities undertaken by SAHRA.

Through the course of the NAMM, 268 individuals benefitted from the employment opportunities available; and some 1785 recordings were captured and submitted at the time of project closure on 30 April 2021, 1366 of which were approved by the Fieldwork Coordinators for import into the national inventory.

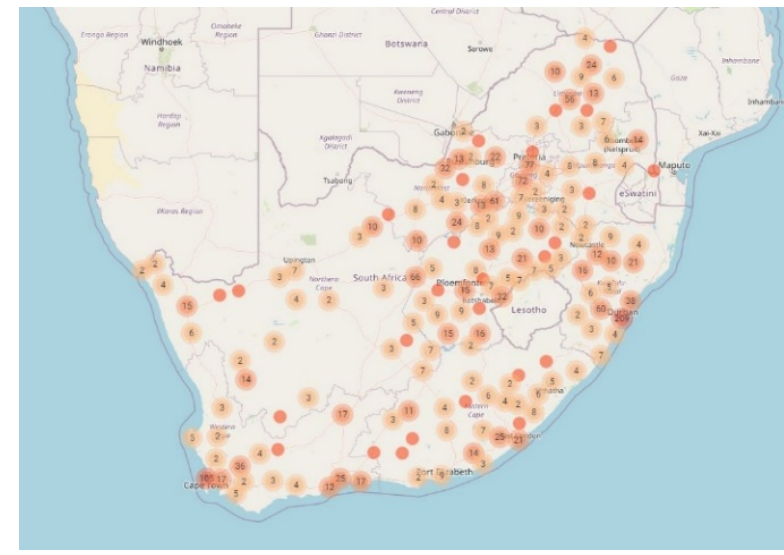

Figure 9: Map of all recordings submitted to KoBoToolbox

After an extensive review and accounting for the challenges noted in the below discussion, the total accepted population was reduced to a final population of 1153 inclusive of those where records existed in the inventory but not further recorded during the NAMM.

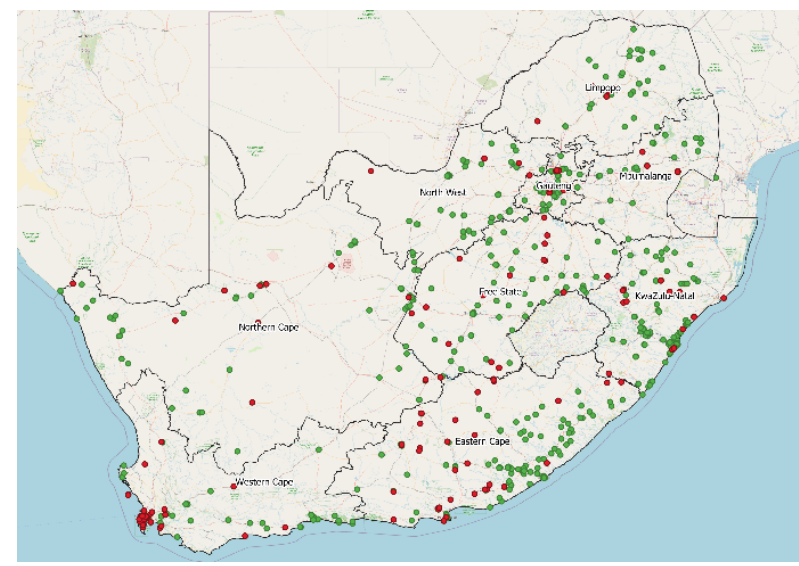

Figure 10: Map of accepted recordings (green) and records, whilst accepted, should be subject to further recording (red).

\subsection{Challenges and Lessons Learned}

The entire project was a learning curve from inception to execution. Firstly, the planning and coordination process heavily relied on inter-governmental interaction with provinces, 
and local authorities. As a result, the processes were not always clear; and in cases where clarity was clear cut, the approval process took longer due to differences in policies. Consequently, the project fieldwork started at varying timeframes across provinces which further necessitated the refinement of the project plan as the project rolled out.

With constrained timelines, adequate training was unattainable. Surveyors and co-ordinators had to be exposed to entirely new systems, in some cases new subject matter, within short periods. As such retraining/refreshing had to be regularly held to address issues as they were identified. A potential pitfall of limited training noted by Dauenhauer et al. (2019) is the input of random data into fields as a coping mechanism to move through the collection form and bypass information prompts that are perhaps not well enough understood. This pitfall was indeed encountered through this project and has resulted in the need to review and possibly re-record certain sites.

After close of the project, it was discovered that within KoBoToolbox, if a record was edited by a Fieldwork Coordinator, the unique ID assigned to each recording would be updated. Whilst it was the intention for the Fieldwork Coordinators to make minor corrections to data prior to approval, the system would not lock out the Fieldwork Coordinator from performing further edits to the record after it was validated. The change in ID of the record after editing resulted in the automated transfer system considering the record to be new and thus was imported onto SAHRIS as a new record, resulting in duplication and the need for comparison between the two versions to decide on retention.

As noted in the discussion on the legal standing of monuments \& memorials in South Africa, the confusion between the previously used term "National Monuments" under now defunct legislation and the modern definition of "Public Monuments and Memorials" resulted in many former National Monuments (now Provincial Heritage Sites) being recorded. Whilst this is useful data for the purposes of the inventory, it will require a period of rationalisation to ensure it is properly incorporated into the existing record for these sites.

\subsection{Project Recommendations}

5.3.1 Data verification: Even though the sites were physically recorded, there is a need to undertake verification by trained heritage practitioners as the data was collection by teams mostly with limited/first time exposure to heritage.

5.3.2 Development of the criteria for categorising the resources: Application or use of the data collected in this project for purposes of heritage landscape transformation requires that a well consulted criteria is developed for determining which of the resources are to be considered as part of effecting the needed transformation.

5.3.3 Establishing the capacity for ongoing research on the associated history of the sites: To ensure correctness and reliability of the data, there is a need for undertake further research on an ongoing basis to ensure that any decisions taken concerning these resources are informed by credible information.
5.3.4 Continuous skills development in heritage management: The young people that participated in this project demonstrated great appreciation of the South African heritage. That showed a great need to expand the capacity of the heritage sector in development of a young, professional body.

\section{CONCLUSION}

The National Audit of Monuments and Memorials presented a unique challenge in terms of project management, heritage management practise, and data collection. Within SAHRA's 21year history, a project of this scope and magnitude had not been attempted, it challenged the project team to reassess how data collection methodology had been planned and implemented previously.

This paper is aimed at discussing the tools used to facilitate a project of this nature, and whether they were successful in their intent to produce rapid results and streamline the process of data capture, review, and inclusion in the inventory of the national estate. Whilst challenges where experienced in the arena of data collection, ultimately the chosen tools and workflows produced positive results within an extremely short space of time. Having now undertaken the groundwork to implement the ecosystem described in this paper, we are convinced that similar projects can utilise rapidly tailored methodologies to deploy any further large (or small) scale recording projects with the benefit of lessons learnt.

\section{REFERENCES}

Loola Bokonda, P., Ouazzani-Touhami, K., \& Souissi, N., 2020. A Practical Analysis of Mobile Data Collection

Apps. International Journal Of Interactive Mobile Technologies (IJIM), 14(13), pp. 19-35.

http://dx.doi.org/10.3991/ijim.v14i13.13483.

Dauenhauer, P., Shields, M., McLean Sloughter, J., Stewart, A. J., Lacrampe, C., Magness, E., Ochavillo, J., Limfueco, J., \& Mendoza, A., 2019. Improving Shoestring Surveys for Off-Grid Humanitarian Power Projects: Kilowatts for Humanity and KoboCollect. In 2018 IEEE Global Humanitarian Technology Conference (GHTC), pp. 1-6.

https://doi.org/10.1109/GHTC.2018.8601657

Department of Arts and Culture, South Africa, 1999. National Heritage Resources Act 25 of 1999. Government Gazette, 506(19974), Pretoria, Government Printers.

Free State Provincial Heritage Resources Authority, 2016. Audit of Statues and Monuments in the Free State. Bloemfontein: Free State Provincial Government.

Heritage Western Cape, 2015. DRAFT GUIDELINES FOR PUBLIC MONUMENTS AND MEMORIALS. Cape Town: Western Cape Provincial Government.

Jackson, C., Mofutsanyana, L., and Mlungwana, N., 2019. A Risk Based Approach to Heritage Management In South Africa, International Archives of the Photogrammetry, Remote Sensing \& Spatial Information Sciences, XLII-2/W15, 591-597, https://doi.org/10.5194/isprs-archives-XLII-2-W15-591-2019.

Kiewit, L. 2020. Pressure to remove colonial relics grows [online]. Mail \& Guardian, 18 June. Available at: 
$<$ https://mg.co.za/news/2020-06-18-pressure-to-removecolonial-relics-grows/> (Accessed: 22 February 2021).

KoBoToolbox. n.d.-a. Features [online]. Available at: <https://www.kobotoolbox.org/\#features> (Accessed: 22 February 2021).

KoBoToolbox. n.d.-b. Editing Forms in Excel using XLSForm [online]. Available at:

<https://support.kobotoolbox.org/edit_forms_excel.html> (Accessed: 29 June 2021).

KoBoToolbox. n.d.-c. Overview of All Formbuilder Functions [online]. Available at:

$<$ https://support.kobotoolbox.org/formbuilder.html> (Accessed: 29 June 2021).

KoBoToolbox. n.d.-d Clone of ODK Collect for use in KoBoToolbox [online]. Available at: $<$ https://github.com/kobotoolbox/collect> (Accessed: 29 June 2021).

KoBoToolbox. n.d.-e. Deploy questionnaires without noting password [online]. Available at:

$<$ https://community.kobotoolbox.org/t/deploy-questionnaireswithout-noting-password/16162/10> (Accessed: 29 June 2021).

KoBoToolbox. n.d.-f. Mapping, Sharing, and Exporting GPS

Data [online]. Available at:

<https://support.kobotoolbox.org/export_gps.html> (Accessed: 29 June 2021).

KoBoToolbox. n.d.-g. Managing Permissions [online].

Available at:

<https://support.kobotoolbox.org/managing_permissions.html> (Accessed: 29 June 2021).

KoBoToolbox. n.d.-h. Using the API [online]. Available at: $<$ https://support.kobotoolbox.org/api.html> (Accessed: 29 June 2021).

Marschall, S., 2006. Transforming the Landscape of Memory: The South African Commerative Effort in Internal Perspective. South African Historical Journal, 55, pp 165-185

Marschall, S., 2017. Monuments and Affordance. Chaiens \& Etudes Africaines, 57 (227), PP 671-690

O’Donoghue, B., 2009. Audit of Sculptures, Memorials and Monuments. Cape Town.

Rankin, E., 2013. Creating/Curating Cultural Capital: Monuments and Museum for Post-Apartheid South Africa. Humanities, 7(2), pp. 72-98

Republic of South Africa. 2020. BUILDING A SOCIETY THAT WORKS: Public investment in a mass employment strategy to build a new economy. Pretoria: The Presidency.

Wiltshire, N., 2013. The use of SAHRIS as a state sponsored digital heritage repository and management system in South Africa. In: ISPRS Annals of the Photogrammetry, Remote Sensing and Spatial Information Sciences, Vol. II-5/W1, pp. 325-330, doi.org/10.5194/isprsannals-II-5-W1-325-2013. 